\title{
Desertificação: uma visão geral dos processos e conceitos, fundamentados em aplicação de índices orbitais através do sensoriamento remoto
}

\author{
Desertification: an overview of processes and concepts, based on the application of orbital indices
}

through remote sensing

Desertificación: una visión general de los procesos y conceptos, basada en la aplicación de índices orbitales a través de la teledetección

Recebido: 27/08/2021 | Revisado: 08/09/2021 | Aceito: 08/09/2021 | Publicado: 11/09/2021

\author{
José Francisco da Cruz Neto \\ ORCID: https://orcid.org/0000-0002-4889-1282 \\ Universidade Federal Rural de Pernambuco, Brasil \\ E-mail: zenetto.agronomia@gmail.com \\ Alexandre Maniçoba da Rosa Ferraz Jardim \\ ORCID: https://orcid.org/0000-0001-7094-3635 \\ Universidade Federal Rural de Pernambuco, Brasil \\ E-mail: alexandremrfj@gmail.com \\ Luciana Sandra Bastos de Souza \\ ORCID: https://orcid.org/0000-0001-8870-0295 \\ Universidade Federal Rural de Pernambuco, Brasil \\ Unidade Acadêmica de Serra Talhada, Brasil \\ E-mail: sanddrabastos@yahoo.com.br \\ Thieres George Freire da Silva \\ ORCID: https://orcid.org/0000-0002-8355-4935 \\ Universidade Federal Rural de Pernambuco, Brasil \\ Unidade Acadêmica de Serra Talhada, Brasil \\ E-mail: thigeoprofissional@ hotmail.com.br
}

\section{Resumo}

A desertificação tem sido um problema ambiental em todo o mundo, em decorrência disso tem se observado nos últimos 50 anos um interesse crescente em relação aos impactos causados na sociedade e na sustentabilidade em geral. A aplicação de técnicas eficientes com ferramentas de baixo custo, é uma alternativa na identificação de áreas em processo de desertificação. Dentre essas técnicas, o sensoriamento remoto vem ganhando destaque devido a sua robustez e base de dados confiáveis. Neste contexto, o objetivo desta revisão é apresentar os principais fatores que influenciam o processo de desertificação em áreas semiáridas, como também, entender os avanços dos estudos sobre desertificação por meio do uso de técnicas de sensoriamento remoto. Atualmente, muitos são os métodos utilizados para identificar áreas desertificadas ou em processo de desertificação. O uso dos índices de vegetação e de solo são fundamentais para identificação dessas áreas, visto que a ausência de cobertura vegetal e o estado do solo podem caracterizar um ambiente desertificado. O sensoriamento remoto tem auxiliado a realização de estudos para grandes áreas e representa uma alternativa promissora para o desenvolvimento de novas metodologias que possam ser utilizadas para a avaliação do risco de desertificação. Os achados desta revisão encorajam a realização de estudos e desenvolvimento de metodologias para estudo de áreas em desertificação especialmente em regiões semiáridas.

Palavras-chave: Degradação; Semiárido; Mudanças climáticas; Cobertura vegetal.

\begin{abstract}
Desertification has been an environmental problem all over the world, as a result of which there has been a growing interest in the last 50 years in relation to the impacts caused on society and on sustainability in general. The application of efficient techniques with low-cost tools is an alternative in identifying areas in the process of desertification. Among these techniques, remote sensing has gained prominence due to its robustness and reliable database. In this context, the objective of this review is to present the main factors that influence the desertification process in semiarid areas, as well as to understand the advances in studies on desertification through the use of remote sensing techniques. Currently, there are many methods used to identify desertified areas or areas in the process of desertification. The use of vegetation and soil indices are essential to identify these areas, as the absence of vegetation cover and soil condition can characterize a desertified environment. Remote sensing has helped to carry out studies for large areas and represents a promising alternative for the development of new methodologies that can be used to assess the risk of desertification. The findings of this review encourage the carrying out of studies and development of methodologies for studying desertified areas, especially in semiarid regions.
\end{abstract}

Keywords: Degradation; Semi-arid; Climate change; Vegetal cover. 


\begin{abstract}
Resumen
La desertificación ha sido un problema ambiental en todo el mundo, por lo que ha habido un creciente interés en los últimos 50 años en relación a los impactos que provoca en la sociedad y en la sostenibilidad en general. La aplicación de técnicas eficientes con herramientas de bajo costo es una alternativa para identificar áreas en proceso de desertificación. Entre estas técnicas, la teledetección ha ganado prominencia debido a su robustez y base de datos confiable. En este contexto, el objetivo de esta revisión es presentar los principales factores que influyen en el proceso de desertificación en las zonas semiáridas, así como comprender los avances en los estudios sobre desertificación mediante el uso de técnicas de teledetección. Actualmente, existen muchos métodos para identificar áreas desertificadas o áreas en proceso de desertificación. El uso de índices de vegetación y suelo es esencial para identificar estas áreas, ya que la ausencia de cobertura vegetal y las condiciones del suelo pueden caracterizar un ambiente desertificado. La teledetección ha ayudado a realizar estudios para grandes áreas y representa una alternativa prometedora para el desarrollo de nuevas metodologías que se pueden utilizar para evaluar el riesgo de desertificación. Los hallazgos de esta revisión fomentan la realización de estudios y el desarrollo de metodologías para el estudio de áreas desertificadas, especialmente en regiones semiáridas.
\end{abstract}

Palabras clave: Degradación; Semiárido; Cambio climático; Cobertura vegetal.

\title{
1. Introdução
}

Os problemas ambientais e socioeconômicos causados pela desertificação têm sido recorrentes em todo o mundo e desencadeado um interesse crescente na realização de estudos nos últimos 50 anos. A desertificação é uma das ameaças globais mais complexas, e é caracterizada como a degradação da terra em áreas áridas, semiáridas e subúmidas secas, resultantes de vários fatores, incluindo variações climáticas e atividades humanas (UNCCD, 1995; Akbari et al., 2020; Ding \& Xingming, 2021; Jardim et al., 2021a), visto que, mais de 40\% de todo o planeta sofre com impactos desse fenômeno (Ding \& Xingming, 2021). Segundo Briassoulis (2019), a desertificação atualmente afeta mais de um terço das terras do mundo, atingindo países como China, Itália, África do Sul, Estados Unidos da América do Norte, México, Argentina e Brasil.

No Brasil, a desertificação encontra-se presente de forma expressiva na região semiárida, com ocorrência observada em 65\% do Nordeste brasileiro (Barbosa Neto et al., 2021). Os estados do Rio Grande do Norte, Pernambuco, Paraíba, Piauí, Ceará e Bahia, possuem grandes extensões degradadas pela desertificação, e foram classificadas como Núcleos de Desertificação (ND) (Vasconcelos Sobrinho, 1982). Os ND tratam-se de áreas de grandes manchas desnudas, com presença ou não de vegetação e sinais claros de erosão do solo (Perez-Marin et al., 2012; Jardim et al., 2017). Seis áreas são caracterizadas como ND: Núcleo de Gilbués - PI; Núcleo de Irauçuba - CE; Núcleo de Cabrobó - PE; Núcleo de Seridó - RN; Núcleo Cariris Velhos - PB e Núcleo do Sertão do São Francisco - BA, que ocupam uma área de 55.000 km² (Briassoulis, 2019).

Nesses ambientes as consequências decorrentes do processo de desertificação são diversas, podendo estar relacionadas as condições ambientais ou mesmo às questões sociais. A desertificação promove perda da fertilidade dos solos, diminuição dos recursos hídricos, redução da biodiversidade, perdas na produtividade agrícola e biológica; e abandono das terras devido à incapacidade de produção de elementos essenciais para a sobrevivência humana. Esses efeitos não trazem implicações apenas aos aspectos ambientais, mas também a setores produtivos da sociedade (Vieira et al., 2015; Sá et al., 2021; Jardim et al., 2021b).

Contudo, muitas controversas surgiram a respeito das causas da desertificação, isso porque existem padrões diferentes para seu surgimento. No Brasil, por exemplo, sua origem em algumas regiões está relacionada ao mal uso dos recursos naturais, como o desmatamento para o uso de madeira como fonte de energia, construções de cercas e casas, a irrigação como suprimento de água para as culturas agrícolas e a falta de manejo correto dos solo (Vasconcelos Sobrinho, 1982; Barros et al., 2018; Jardim et al., 2021c), isso tudo é agravado devido as condições climáticas da região, que apresenta uma alta demanda atmosférica e grande variabilidade da precipitação (Mariano et al., 2018; Silva et al., 2020a; Santos et al., 2020; Silva et al., 2021a). Neste contexto, o monitoramento e a avaliação das causas e consequências da desertificação são extrema relevância para os países e ecossistemas. Para tanto, sua avaliação criteriosa e precisa da desertificação pode fornecer uma base científica para a tomada de decisões no combate/minimização dos processos degradativos. Desta forma, devido a grandes quantidades de 
informações, ciclos curto e amplo banco de dados, menos restrições aos recursos humanos, materiais e assim por diante, o sensoriamento remoto tornou-se uma tecnologia importante para monitorar a desertificação da terra nos últimos 30 anos (Wei et al., 2018; Mariano et al., 2018; Fathizad et al., 2018; Costa et al., 2021).

Pesquisas são desenvolvidas para identificar, quantificar e mitigar os efeitos da desertificação, por meio de metodologias e indicadores utilizando técnicas de Sensoriamento Remoto. Algumas são mais recentes outras mais antigas, mas em ambos os casos, sempre se busca maior precisão para monitoramento do avanço do processo de desertificação (Oikonomou et al., 2019; Xue et al., 2019; Briassoulis, 2019; Akbari et al., 2020; Karavitis et al., 2020). Em um estudo para analisar a desertificação no Platô, Wei et al. (2018) desenvolveram modelos com uso do NDVI (Normalized Difference Vegetation Index), Albedo (albedo da superfície da terra), MSAVI (Modified Soil Adjusted Vegetation Index) e TGSI (Topsoil Grain Size Index) e observaram que estes conseguiram explicar em média 86,33\% dos efeitos da desertificação nessa região. Além disso, os autores constataram que o modelo que leva em consideração Albedo-NDVI, apresentam boa precisão para regiões onde a cobertura vegetal é de grande proporção.

No geral, muitos métodos são questionados, por causa da necessidade de numerosa quantidade de variáveis, outras por serem restritas a alguns locais e requererem calibração quando usados em condições distintas das quais foi desenvolvido; isso acaba na maioria das vezes comprometendo os resultados obtidos para essas regiões, o que torna necessário estudos dessa natureza para esses ambientes. Um trabalho que é tido como referência para os estudos sobre desertificação é o de Kosmas et al. (1999), que usa índices na identificação de áreas desertificadas e seus efeitos na região do Mediterrâneo. Mesmo mostrando resultados significativos e representando bem as áreas desertificadas, esse modelo esbarra na grande quantidade de variáveis utilizadas, o que de certa forma pode inviabilizar a sua utilização. Assim, metodologias que empreguem menor quantidade de variáveis e boa eficiência na estimativa devem ser desenvolvidas para permitir aplicação em diferentes condições e escalas temporais. O objetivo desta revisão é apresentar os principais fatores que influenciam o processo de desertificação em áreas semiáridas, como também, entender os avanços dos estudos sobre desertificação por meio do uso de técnicas de sensoriamento remoto.

\section{Metodologia}

No presente trabalho, nós confeccionamos uma revisão sistemática fundamentada em trabalhos científicos indexados em bases de dados eletrônicas como ScienceDirect, Scopus e Google Scholar sem restrição de idiomas, baseando-se em materiais com significativa relevância científica em ciências agrárias e ambientais (Silva et al., 2020b; Xavier Junior et al., 2021). Por se tratar de uma temática bastante ampla, a mineração dos trabalhos não se limitou à estudos da última década. Utilizamos como estratégia na busca do material científico para compor a revisão termos de indexação e palavras-chave como: água, desertification, risk, climate changes, pastagem, degradação, semi-arid, Northeast Brazil, low rainfall, dry, NDVI, EVI, environmental, drought risk, index, global, integrating vegetation, soil, climate and anthropic indices.

\section{Revisão da Literatura}

\subsection{Histórico da desertificação}

A desertificação é um problema de escala global, que tem tomado notoriedade, por causa da degradação das terras promovida pela ação humana e mudanças climáticas, sobretudo, em regiões de clima árido, semiáridos e subúmidas a secas (Kosmas et al., 1999; Sommer et al., 2011). Os primeiros registros foram feitos na década de 30, nos estados do meio Oeste Americano, onde se observaram intensos processos de degradação (e.g. aumento do desmatamento, exploração acentuada do solo e dos recursos naturais, seguido de uma grande seca entre os anos de 1929 e 1932). Nessa época, estimou-se que 300.000 $\mathrm{km}^{2}$ das terras foram devastadas nos estados de Oklahoma, Kansas, Novo México e Colorado, com subsequente perda 
econômica (Stebbing, 1935; Nippert et al., 2013).

Apesar desse registro, o termo desertificação foi proposto pela primeira vez em 1949 por Aubréville, o qual foi usado para caracterizar processos ecológicos ocorridos na África Tropical, em especial, alterações de florestas tropicais em áreas com pouca vegetação. Mas, ao longo dos anos, muitas situações ocorreram em diferentes regiões do mundo. Por exemplo, na década de 60, registrou-se em regiões da África, a perda da capacidade produtiva dos solos e a falta de alimentos, seguida, de fome, seca e guerra, o que culminaram em incremento do fluxo migratório na década de 70 (Aubréville, 1949; Glantz, 1977; Ferreira et al., 2017; Barros Corrêa et al., 2019) de regiões áridas e semiáridas ao Sul do deserto do Sahara, conhecida como Sahel (Glantz, 1977).

No Brasil, os primeiros estudos de desertificação foram feitos para o Semiárido por João Vasconcelos Sobrinho da Universidade Federal Rural de Pernambuco, que no ano de 1974 publicou o artigo "O Deserto Brasileiro". Nesta obra, Sobrinho indicou os "Núcleos de Desertificação", localizados na microrregião do Sertão de Inhamuns, Ceará; no município de Gilbués, Piauí; na região do Seridó, Rio Grande do Norte; região dos Cariris Velhos, Paraíba; Sertão Central do estado de Pernambuco; e, no Sertão do São Francisco, Bahia, onde as degradações da cobertura vegetal e do solo atingiram condição de irreversibilidade (Vasconcelos, 2004; Mariano et al., 2018).

Atualmente, a desertificação é um dos principais problemas ambientais, que promovem prejuízos econômicos e sociais (Briassoulis, 2019), causando uma perda de 12 milhões de ha por ano (Vieira et al., 2020). Logo, o seu monitoramento é muito importante para proposição de ações mitigadoras. Diante disto, diversas pesquisas (Oikonomou et al., 2019; Xue et al., 2019; Briassoulis, 2019; Akbari et al., 2020; Karavitis et al., 2020) tem desenvolvido metodologias para que se possa quantificar de forma mais eficiente os níveis de desertificação em diferentes regiões do mundo. A desertificação é um processo complexo e representa um campo vasto de estudo. No Brasil, embora alguns métodos já tenham sido aplicados para identificação das áreas em processo de desertificação (Sousa et al., 2007; Fernandes e Dantas, 2010; Lopes e Soares, 2016; Tomasella et al., 2018) mais pesquisas são necessárias para fundamentar melhor sua aplicação e acurácia.

\subsection{Conceitos de desertificação}

Desertificação é um termo que deriva da etologia “Deserto". Segundo Conti (2008), o deserto é uma formação natural que ocorre como resultado de fatores climáticos (i.e., evaporação potencial maior que a precipitação média anual, e precipitação escassa com alta variabilidade anual) combinados a solos rasos; drenagem intermitente; cobertura vegetal esparsa com o predomínio de espécies xerófilas e baixo índice de ocupação humana. Ao conceito de desertificação, adiciona-se o efeito da ação humana, o que potencializa os danos não só ao meio ambiente, mas também as populações que habitam nessas regiões.

Diversos autores classificam a desertificação com base em fatores humanos, ao tempo geológico, forma de relevo, vegetação e aspectos de impactos econômicos (Verstraete, 1986). Do ponto de vista da ação humana, Gardufio (1977) e Bezerra et al. (2020) citam que a desertificação é o empobrecimento de ecossistemas áridos, semiáridos e subúmidos a seco; maximizado pela variabilidade climática. Quanto ao tempo geológico, a desertificação é como desertos naturais que estão distribuídos pela superfície da Terra, e cuja existência flutuou no período quaternário, presentes em antigos prados, pradarias, estepes e planícies aluviais. Le Houérou (1977) enfatizou a desertificação como um processo que envolve o relevo e a vegetação, caracterizando-a como uma extensão de paisagem desértica típica, com degradação de vários tipos e formas de vegetação, incluindo as florestas subúmidas. Por sua vez, as Nações Unidas, em 1978, definiram a desertificação como a diminuição do potencial biológico do solo, a produtividade dos ecossistemas.

Como pioneiro no Brasil, Vasconcelos Sobrinho (1982) considera a desertificação como um fenômeno integrador de processos econômicos, sociais e naturais, que ocasiona desequilíbrio do solo, da vegetação, do ar e da água, bem como a 
qualidade da vida humana. Para Vasconcelos Sobrinho (1982), a degradação da interface solo, vegetação e recursos hídricos, resultado de condições climáticas, ações humanas, ou de ambas, leva a redução da produtividade primária e perda da capacidade de auto recuperação do solo. Rodrigues (1992) complementa o conceito de Vasconcelos Sobrinho, afirmando que a desertificação pode ser intensificada por atividades como desmatamento, extração de minério, uso inadequado da irrigação e de fertilizantes químicos na produção de alimentos, e sobrepastejo. Retallack (1991) entende que a desertificação rompe a estabilidade de uma paisagem, dando origem a eventos de erosão, denudação de solos cultiváveis, formação de campos de dunas, e tornam esses ambientes inapropriados para o desenvolvimento de atividades agrícolas e de sobrevivência humana.

Apesar dos diversos conceitos, um comumente adotado é que a desertificação refere-se a uma sequência de alterações regressivas dos solos, da vegetação e do regime hídrico, que conduz à deterioração biológica dos ecossistemas, em consequência de pressões criadas por fatores climáticos, e pelas atividades do homem, em ações conjuntas ou separadas (CGEE, 2016).

\subsection{Panorama da desertificação no Semiárido brasileiro}

No Brasil, a desertificação ocorre de forma restrita no Semiárido brasileiro, além disso, a região Nordeste é caracterizada como local de maiores problemas degradativos e áreas com processo de desertificação (Bezerra et al., 2020). Com base no estudo de Vasconcelos Sobrinho (1982), para o Semiárido brasileiro (SB), seis áreas pilotos exibem processos de degradação do solo e da cobertura vegetal. Essas áreas estão situadas nos estados do Rio Grande do Norte, Pernambuco, Paraíba, Piauí, Ceará e Bahia, e os seus limites são usados pelo Ministério do Meio Ambiente como aquelas áreas mais sujeitas à degradação ambiental por meio da desertificação. Essas áreas, denominadas "Núcleos de Desertificação" possuem grandes manchas desnudas, com presença ou não de vegetação e sinais claros de erosão do solo (Perez-Marin et al., 2012; Bezerra et al., 2020) (Figura 1). No semiárido brasileiro há diversas áreas com os mesmos aspectos encontrados nesses Núcleos, mas a falta de metodologias adequadas ainda não as enquadra como áreas desertificadas ou que estejam enfrentando esse processo. Abaixo são descritos os principais atributos dos "Núcleos de Desertificação" presentes no Semiárido brasileiro. 


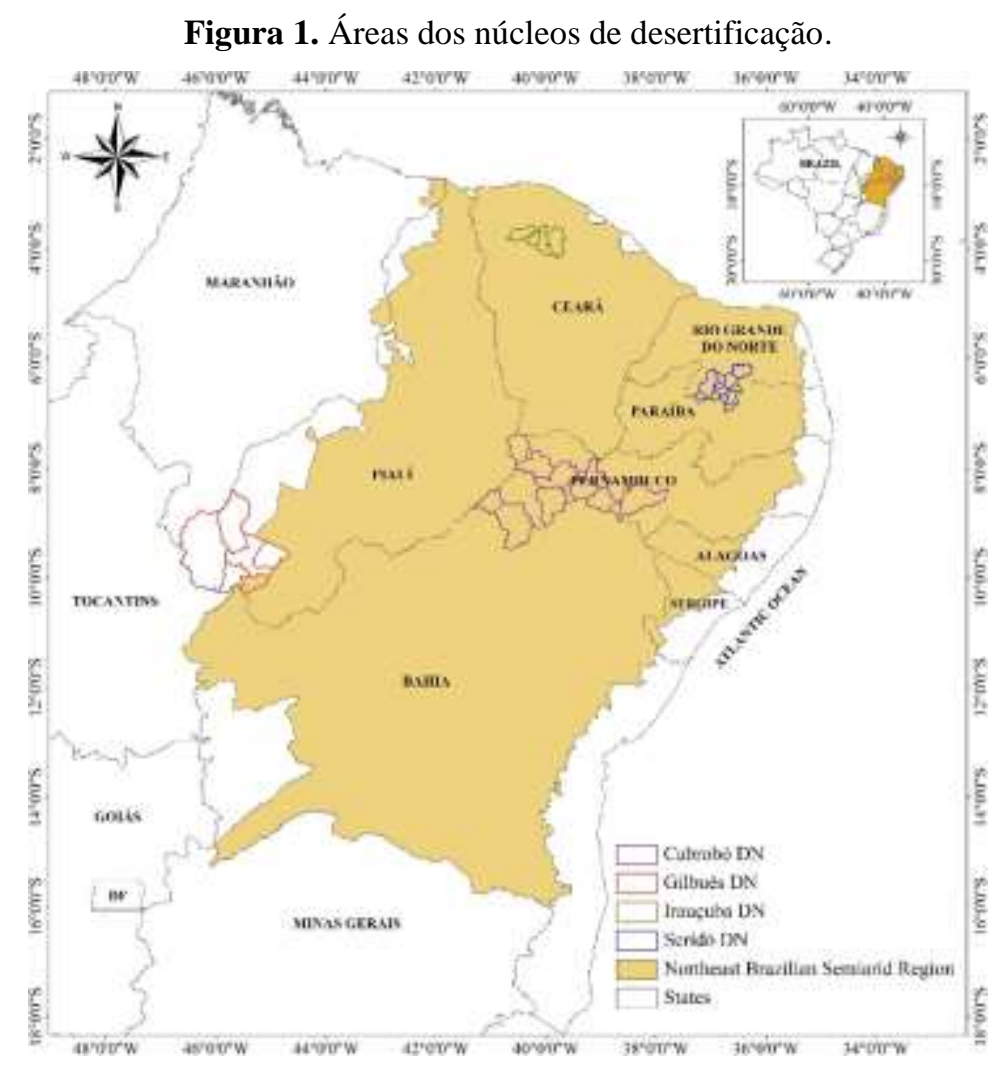

Fonte: Bezerra et al. (2020).

\section{Núcleo de Gilbués}

Localizado no extremo Sul do estado do Piauí, na região de transição com os biomas de Caatinga e Cerrado (Figura 1), compreende área afetada de $6.131 \mathrm{~km}^{2}$, com 20 mil habitantes (Perez-Marin et al., 2012). É composto pelos municípios de Gilbués, Monte Alegre do Piauí, Barreiras do Piauí, São Gonçalo do Gurgueia, Santa Filomena e Alto Parnaíba. Nesse núcleo, a desertificação está ligada aos processos erosivos dos solos. As principais ações que contribuem para esse processo ocorrem devido à grande exploração da pecuária, e retirada da vegetação nativa para implantação de culturas que são utilizadas na alimentação animal, como também a produção de carvão vegetal.

\section{Núcleo de Irauçuba}

Situado no Oeste do estado do Ceará, região dos Inhamuns, compreende área afetada de 4.099,22 km² dos municípios de Sobral, Forquilha e Irauçuba (Pinto et al., 2009) (Figura 1). A principal caracterização desse núcleo está relacionada a categoria de solo presente (Planossolos háplicos com caráter sódicos e nátricos), a grande ocorrência de erosão laminar avançando para voçorocas (proveniente das chuvas e solos expostos), o que empobrece o solo e impossibilita a restituição vegetal da região. Os processos de erosão neste núcleo estão intrinsecamente ligados aos fatores edáficos, logo que, por apresentarem solos com baixa profundidade e capacidade de infiltração, existe um favorecimento à ocorrência de erosão, principalmente laminar. Somadas as práticas errôneas, e cultivos intensivos de monoculturas, a degradação ambiental deste núcleo eleva sua magnitude (Sá \& Angelotti, 2009).

\section{Núcleo de Cabrobó}

Localizado no Sul do Estado de Pernambuco, região do Sertão Central, onde estão os municípios de Cabrobó, Orocó, Santa Maria da Boa Vista, Belém do São Francisco, Salgueiro, Parnamirim, Itacuruba, Petrolina, Afrânio, Ouricuri, Araripina e Floresta (Figura 1). A área afetada é de $4.960 \mathrm{~km}^{2}$ (Perez-Marin et al., 2012). Silva et al. (2015) relata que a salinização, 
devido ao mau uso da irrigação, a pecuária e o desmatamento são as maiores causas do processo de desertificação. Essas afirmações também foram identificadas por Sá \& Angelotti (2008). Outro fator agravante neste núcleo, é o sobrepastejo das áreas, atividade comum na região, que intensifica os processos degradativos, causa exaurimento do solo, e compactação das camadas superficiais, impedindo a ciclagem de nutrientes (Sales, 2006; Jardim et al., 2018).

\section{Núcleo de Seridó}

Situado em parte dos Estados do Rio Grande do Norte - RN e da Paraíba - PB, no Seridó (Figura 1). O Núcleo abrange os municípios de Currais Novos, Cruzeta, Equador, Carnaúba do Dantas, Acari, Parelhas, Caicó, Jardim do Seridó, Ouro Branco, Santana do Seridó e São José do Sabugi no RN, e Santa Luzia e Várzea, na PB. A área afetada é de $2.987 \mathrm{~km}^{2}$ (Perez-Marin et al., 2012). A retirada da lenha para abastecimento das fábricas de cerâmicas que existem na região é considerado o principal fator para o avanço da desertificação na região (Pereira Neto, 2016). Além disso, os baixos índices pluviométricos dificultam a regeneração da vegetação, o que agrava o processo (Perez-Marin et al., 2012).

\section{Núcleo Cariris Velhos}

Localizado na fração voltada ao Sudoeste da Chapada da Borborema paraibana, região dos Cariris Velhos. Abrange os municípios de Juazeirinho, São João do Cariri, Serra Branca, Cabaceiras, Camalaú e municípios circunvizinhos. A área afetada é de $2.805 \mathrm{~km}^{2}$, sobretudo, devido à formação geológica local, baixos índices pluviométricos, desmatamentos e uso inadequado de práticas agrícolas que empobrecem os solos, tornando-os mais vulneráveis ao progresso da desertificação (Perez-Marin et al., 2012).

\section{Núcleo do Sertão do São Francisco}

Situado no Nordeste do estado da Bahia, na região do Sertão do São Francisco, compreende uma área afetada de $34.254 \mathrm{~km}^{2}$, que abrange os municípios de Uauá, Macururé, Chorrochó, Abaré, Rodelas, Curaçá, Glória, Jeremoabo, Juazeiro, Canudos, e municípios circunvizinhos (Perez-Marin et al., 2012). Nesse núcleo, o processo de desertificação tem origem mediante a substituição da vegetação nativa "Caatinga" para implantação de atividades agrícola e pecuária, além de práticas ineficientes de uso dos recursos naturais (Silva et al., 2011).

\subsection{Indicadores para identificação da desertificação}

Ao longo dos anos diversos estudos internacionais foram desenvolvidos para entender o processo de desertificação (Olsson et al., 2005; Lin et al., 2009; Albalawi \& Kumar, 2013). De início os principais aspectos levados em consideração eram com relação a degradação dos solos ainda nos anos 70, quando os estudos relacionados a desertificação ainda surgiam (Basso et al., 2000). Com isso foi possível identificar que a forma de se utilizar o solo poderia acelerar ou não esse processo em diversas paisagens.

Um dos estudos pioneiros é o de Reining (1978), que inspirou o primeiro trabalho realizado no Brasil, desenvolvido por Vasconcelos Sobrinho (1982). Este autor listou 34 indicadores, sendo eles divididos em seis categorias: físicos (8 indicadores); biológicos agrícolas (8); uso da terra (6); assentamento das populações (4); biológicos humanos (3); e, processo social (5). Um dos problemas do estudo de Reining (1978) é a falta de informação sobre a mensuração dos dados usados, o que dificulta a replicação do estudo e, ou, aprimoramento do método desenvolvido. É importante salientar que o estudo não levou em consideração o aspecto climático, que é um fator primordial, sobretudo quando se trata da influência do regime pluviométrico no desenvolvimento da vegetação e da promoção de erosão (Vieira et al., 2015). No geral, os indicadores de desertificação são classificados em biológicos, físicos, espectrais e socioeconômicos (Matallo Junior, 2001). 
Em diversos estudos foram utilizados indicadores como albedo, NDVI e SAVI para se identificar o grau de desertificação em que as regiões afetadas se encontravam, mas devido ao grau de processos de desertificação, diferentes texturas de solo (Wei et al., 2018), torna-se inviabilizando muitas vezes a utilização desses indicadores. Dessa forma o indicador utilizado para identificar a degradação do solo em áreas desertificadas é o TGSI (Topsoil Grain Size Index) (Gebru et al., 2021; Qian et al., 2021). Lamchin et al. (2017) descobriram que as maiores correlações estavam entre TGSI e albedo em todos os níveis de desertificação. Isso fornece a hipótese para a construção de um modelo mais eficiente com base no estudo citado. Desta forma, combinando as informações de superfície por medição direta e orbitais, pode-se observar que esses estudos sobre desertificação se baseiam, principalmente, em combinar vários índices ou em um único modelo, o que facilitaria esse tipo de estudo.

Esse TGSI está associado às propriedades físicas (composição estrutural do solo superficial) e baseia-se no diâmetro médio ou efetivo de grãos ou partículas minerais individuais (Xiao et al., 2006). O TGSI reflete o aumento do tamanho do grão do solo superficial, que tem uma correlação positiva com o teor de areia fina do solo superficial. Segundo Lamchin et al. (2017), quanto mais severa a desertificação, mais espesso será o tamanho dos grãos do solo. Valores altos de TGSI são encontrados em áreas com alto teor de areia fina no solo superficial ou baixa proporção de grãos de argila-silte.

De toda forma, variáveis como NDVI e albedo são de fundamental importância para a identificação de ambientes desertificados, visto que estudos a longo prazo mostram que, com o aumento da desertificação, a quantidade de vegetação na superfície do solo diminui de forma gradativa e os valores de NDVI diminuem proporcionalmente (Wei et al., 2018) (Figura 2). O albedo por exemplo é um dos parâmetros mais importantes do balanço energético da radiação no solo e seus valores podem ser alterados pela umidade do solo, cobertura vegetal e outras condições da superfície do solo que podem caracterizar ambientes desertificados.

Figura 2. Diagrama esquemático da análise de vetor com base em valores de NDVI e albedo de superfícies vegetadas a corpos hídricos.

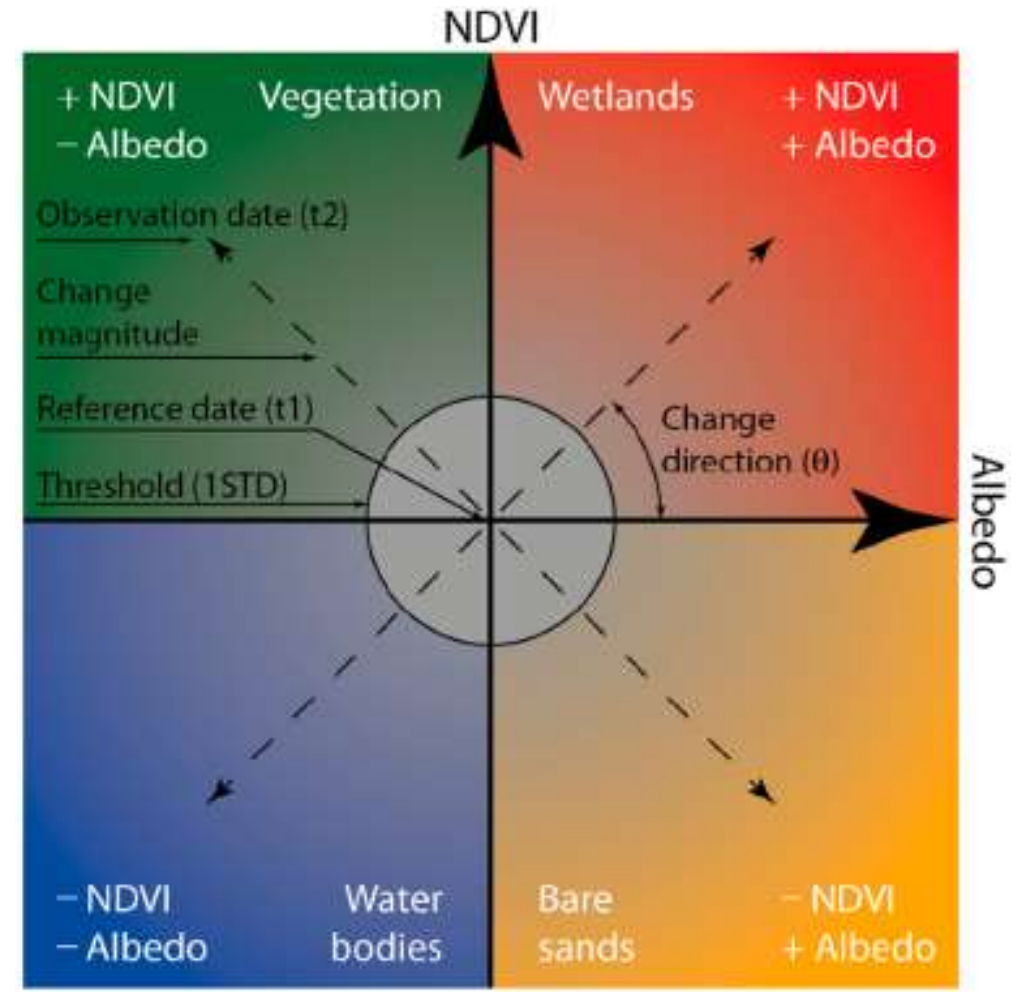

Fonte: Karnieli et al. (2014). 
O índice de aridez (IA) é um dos indicadores mais usados na identificação de áreas desertificadas, ou que tenha potencial para ocorrência desse fenômeno. Segundo Sales (2002), o IA permite conhecer as limitações impostas pelo clima para a realização de atividades biológicas primárias e, logo, na produtividade agrícola de todo o globo terrestre (Figura 3). O IA estabelece a relação entre a precipitação e a evapotranspiração de potencial determinada pelo método de Thornthwaite e foi proposto por UNEP (1991). Salvati et al. (2011) realizaram um estudo para identificar os avanços de áreas degradadas na Itália, utilizando 26 índices de desertificação, dos quais nove são associados ao clima. Em trabalhos mais recentes, Lin et al. (2017) estabeleceu relações entre dados climáticos e imagens de satélites para a identificação de diferentes tipos de superfícies, indicando que esses estudos podem ser úteis na identificação de áreas degradadas.

Figura 3. Visão geral do mapa mundial do índice de aridez da FAO de 1981 a 2010.

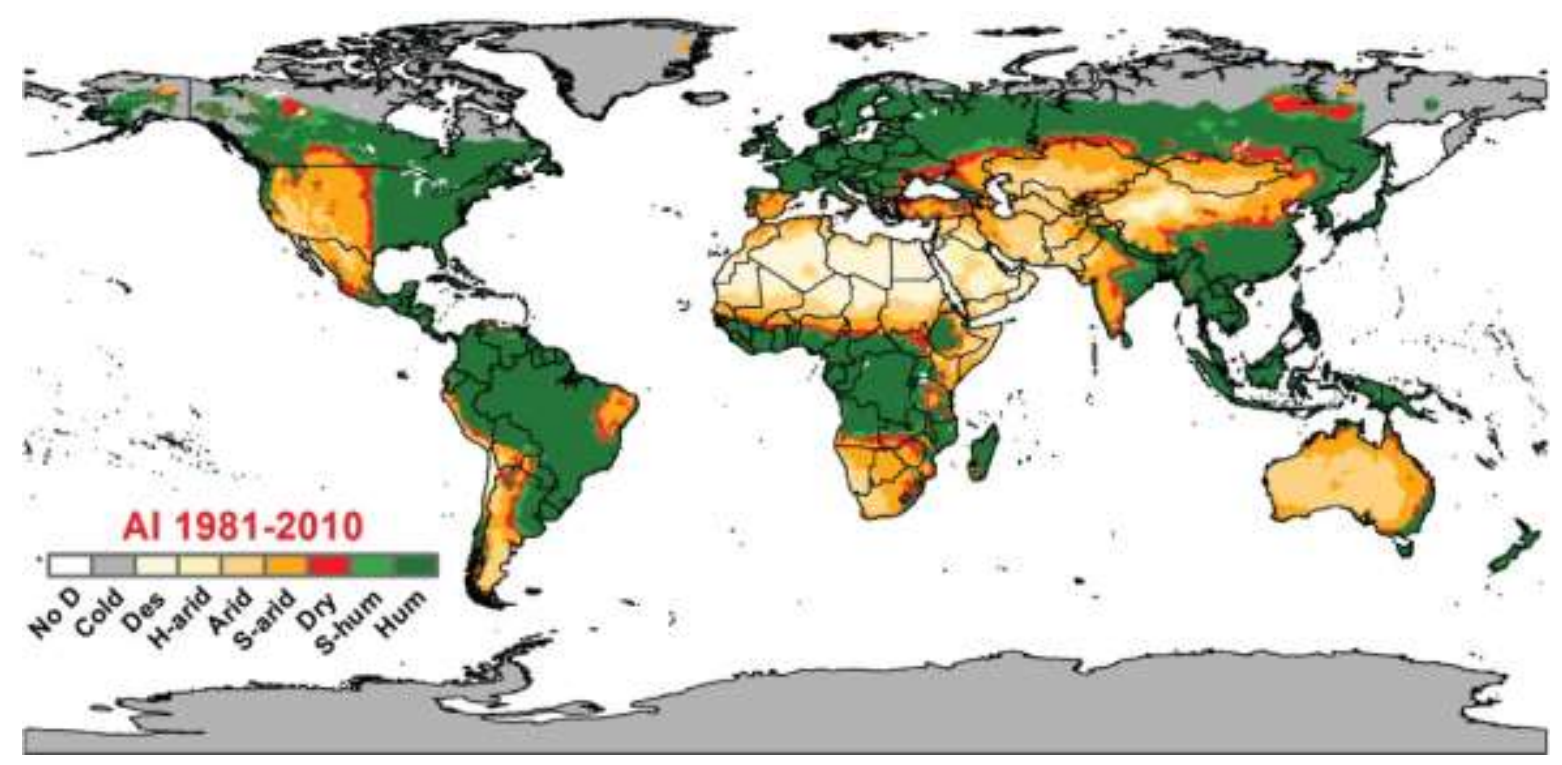

Fonte: Spinoni et al. (2015).

Em conjunto com esses indicadores muitos outros são propostos e utilizados em estudos que caracterizam ambientes desertificados, como nos estudos reportados na literatura (Xiao et al., 2006; Kosmas et al., 2013; Salvati et al., 2016; CGEE, 2016; Akbari et al., 2016; Verón et al., 2018; Joseph et al., 2018; Akbari et al., 2020; Karavitis et al., 2020). Porém, a complexidade e o número grande de variáveis muitas vezes inviabilizam a aplicação dessas metodologias, além de que, o fator micrometeorológico não é levado em consideração bem como, estudos in situ que caracterize melhor o ambiente em estudo.

\subsection{Impactos da desertificação}

As consequências decorrentes do processo de desertificação são diversas, podendo estar associadas a efeitos ambientais ou até a questão social. Um aspecto que tem influenciado diretamente o avanço do processo de desertificação é o aquecimento global que tem elevado à degradação das terras em escala global (Biskaborn et al., 2019), afetando significativamente o sistema vegetação-solo, com perdas de produtividade e capacidade de regeneração dos solos, causando impactos que agravam o processo de desertificação (Reynolds et al., 2011; Feng et al., 2016).

Segundo Araújo et al. (2017), a desertificação leva à redução da produtividade e da diversidade das formas de vida, a degradação acelerada do solo e o aumento dos riscos inerentes à ocupação da terra pelo homem. Atualmente o processo de desertificação atinge cerca de um terço das terras do planeta, atingindo países como Brasil, Estados Unidos da América do Norte, China entre outros.

De forma alternativa, as mudanças nos tipos de vegetação e na densidade causadas pela desertificação impactam a 
interação hidrotérmica entre a atmosfera e o solo (Herb et al., 2008; Yang et al., 2010), e de forma simultânea, o estado térmico do solo afetando intimamente ambientes ecológicos (Shaoling et al., 2000), perdas de carbono orgânico (Crowther et al., 2016; Bai et al., 2019), atividades microbianas (Lavee et al., 1998; Sepehri et al., 2019) e impactam os processos hidrológicos (Cheng \& Wu, 2007; Wu et al., 2018; Chen et al., 2020; Pinheiro et al., 2021). Esses processos afetam diretamente o albedo da superfície (Xie et al., 2015), e consequentemente acarreta na redução da umidade do solo (Teufel \& Sushama, 2019), com efeitos nas propriedades térmicas e hidráulicas do solo (Niu et al., 2019).

O processo de desertificação proporciona e induz a ambientes desérticos, perda da fertilidade dos solos, diminuição dos recursos hídricos, diminuição da biodiversidade, perdas na produtividade agrícola/biológica e abandono das terras devido à incapacidade de produção de elementos essenciais para a sobrevivência humana (Vieira et al., 2015; Vieira et al., 2016; Jardim et al., 2021d). Em termos gerais, as principais origens que promovem desertificação e suas respectivas consequências estão ligados aos elementos solo, clima e atividade antrópica (Figura 4).

Figura 4. Modelo de análise para identificar os caminhos que causam a desertificação.

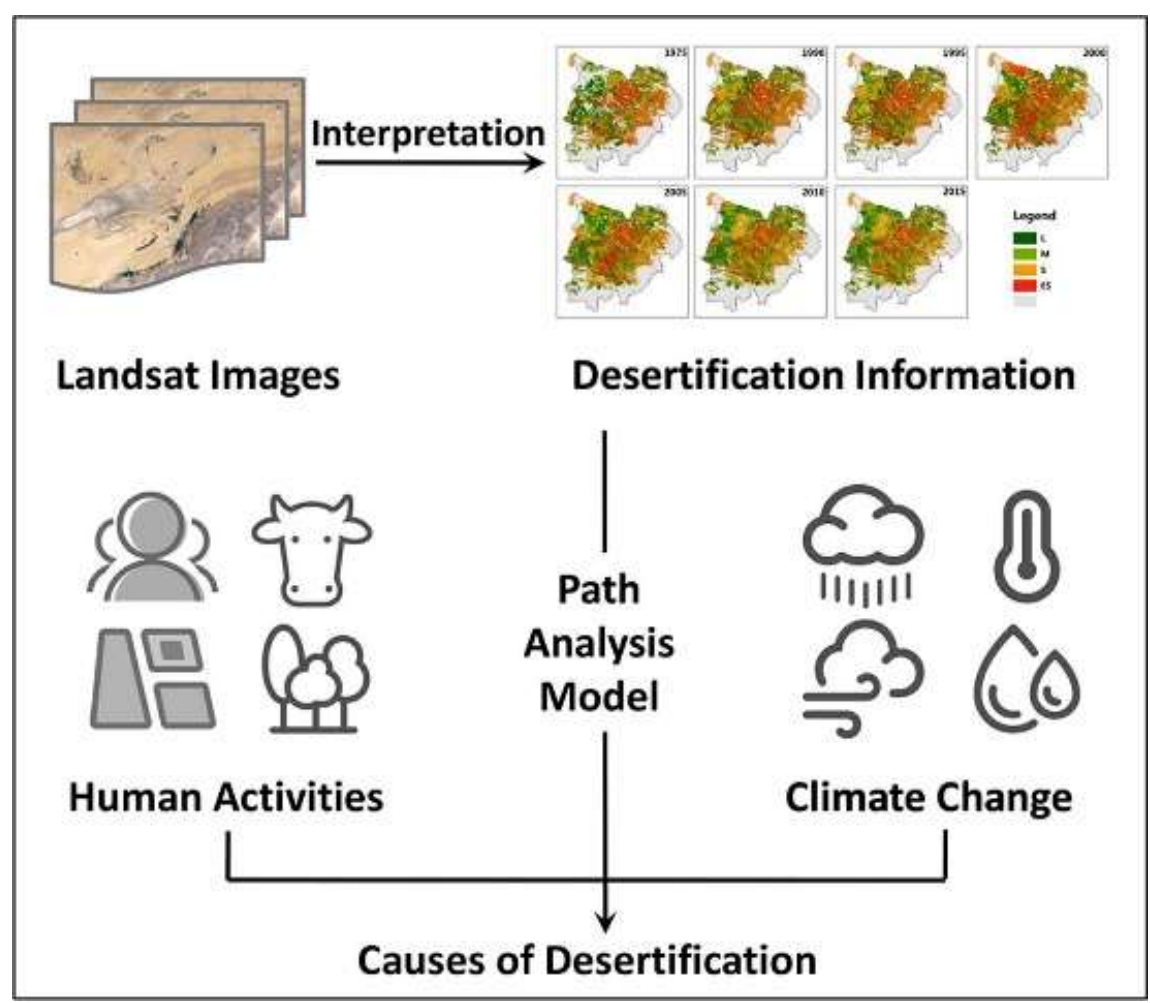

Fonte: Feng et al. (2021).

\subsection{Técnicas de sensoriamento remoto aplicado aos estudos da desertificação}

O monitoramento e avaliação da desertificação são conteúdos muito importantes no contexto desse processo que atinge atualmente e afeta mais de um terço das terras do mundo (Briassoulis, 2019). A avaliação científica e precisa desse fenômeno pode fornecer base científica para a tomada de decisões na mitigação da desertificação. Devido à abundância de informações, ciclo curto e amplo escopo de dados, menos restrições aos recursos humanos e materiais, e assim por diante; o sensoriamento remoto se tornou uma tecnologia importante para monitorar a desertificação da terra nos últimos 30 anos (Pan \& Li, 2013). Dessa forma a avaliação quantitativa dos métodos atuais de sensoriamento remoto para desertificação usados é obtida principalmente através do índice e cobertura vegetal, para obter informações sobre a extensão da desertificação.

A técnica de sensoriamento remoto trata do uso de imagens orbitais como ferramenta para estudos e acompanhamento das mudanças ambientais, sendo atualmente indispensável para análise da variação espaço-temporal de problemas relacionados 
ao meio ambiente. Para estudos relacionados à desertificação, diferentes autores já constatam a eficiência dessa ferramenta (Salvati et al., 2011; Ferreira et al., 2017; Prăvălie et al., 2020), dado que o computo do nível de cobertura vegetal, facilmente obtido por meio do sensoriamento remoto, tem grande associação com a desertificação.

Muitas são as informações que se pode obter por meio do sensoriamento remoto como, por exemplo os índices de vegetação que são informações úteis, pois estabelece relação direta entre dados radiométricos da superfície e a fitomassa foliar verde (Agarez et al., 2001; Nolêto, 2005; Rudorff et al., 2007). Outros indicadores de superfície são: o albedo, FVC (Frational Vegetation Cover), TVDI (Tenperature Vegetation Druyness Index), MSAVI (Modified Soil Adjusted Vegetation Index), TGSI (Topsoil Grain Size Index), PDI (Perpendicular Drought Index), TWI (Topographic Wetness Index) e NDVI (Normalized Difference Vegetation Index) entre outros. De toda forma, estudos desenvolvidos em uma escala de tempo pequena, e sem dados de superfície não são confiáveis para caracterizar ambientes em desertificados (Wei et al., 2018), logo que alterações das informações (e.g., respostas da vegetação à eventos de chuva) em curtos intervalos podem mascarar os resultados obtidos apenas por imagens de satélite.

Por meio desses indicadores, Liu et al. (2005), entre os anos de 1995 e 2001, identificaram variações no avanço da desertificação para a região da Ásia central. Estudos com base em dados de sensoriamento remoto são de fundamental importância, especialmente quando se deseja estudar grandes regiões como é o caso do Semiárido brasileiro (Lin et al., 2017; Silva et al., 2021b), sobretudo, devido à complexidade que existem nos atuais modelos para identificação de áreas em desertificação, o que dificulta a sua avaliação.

\section{Considerações Finais}

A desertificação é um dos principais problemas ambientais no mundo atualmente, e consiste na degradação de terras e perca da capacidade produtiva dessas áreas, contudo, é importante citar que os avanços encontrados nos estudos relacionados a desertificação são bastante válidos, tem contribuído com a identificação dessas áreas, e mesmo, facilitado a busca por soluções que minimizem esses impactos e ajudem a revitalizar esses ambientes. No Brasil, a desertificação se restringe apenas ao Semiárido brasileiro, devido principalmente as condições climáticas da região.

Atualmente, muitos são os métodos utilizados para identificar áreas desertificadas ou em processo de desertificação. Vale salientar, que índices de vegetação e de solo são fundamentais para identificação dessas áreas, visto que a ausência de cobertura vegetal e o estado do solo podem caracterizar um ambiente desertificado. O sensoriamento remoto tem auxiliado a realização de estudos para grandes áreas e representa uma alternativa promissora para o desenvolvimento de novas metodologias que possam ser utilizadas para a avaliação do risco de desertificação.

Os achados desta revisão encorajam a realização de estudos futuros e desenvolvimento de metodologias para estudo de áreas em desertificação especialmente em regiões áridas e semiáridas.

\section{Agradecimentos}

Os autores gostariam de agradecer o apoio financeiro da Coordenação de Aperfeiçoamento de Pessoal de Nível Superior (CAPES - Código de Financiamento 001), Fundação de Amparo à Ciência e Tecnologia do Estado de Pernambuco (FACEPE - APQ-0215-5.01/10 e FACEPE - APQ-1159-1.07/14), e ao Conselho Nacional de Desenvolvimento Científico e Tecnológico (CNPq - 475279/2010-7, 476372/2012-7, 305286/2015-3, 309421/2018-7, e 152251/2018-9) pelo apoio financeiro e concessão das bolsas dos autores. 


\section{Referências}

Agarez, F. V., Vinces, R. S, Cruz, C., M., Nogueira, C., R., \& Garay, I. (2001). Utilização de Índice de vegetação na classificação integrada de fragmentos florestais em Mata Atlântica de Tabuleiros no Município de Sooretama, ES. X Simpósio Brasileiro de Sensoriamento Remoto (SBSR), 955-958.

Akbari, M., Ownegh, M., Asgari, H. R., Sadoddin, A., \& Khosrav, H. (2016). Desertification risk assessment and management program. Global Journal of Environmental Science and Management, 2, 365-380.

Akbari, M., Shalamzari, M. J., Memarian, H., \& Gholami, A. (2020). Monitoring desertification processes using ecological indicators and providing management programs in arid regions of Iran. Ecological Indicators, 111, 106011.

Albalawi, E. K., \& Kumar, L. (2013). Using remote sensing technology to detect, model and map desertification: A review. Journal of Food, Agriculture \& Environment, 11(2), 791-797.

Araújo, J. A., Souza, R. F. (2017). Abordagens sobre o processo de desertificação: uma revisão das evidências no Rio Grande do Norte. Geosul, 32(65), 122143.

Aubréville, A. (1949). Climats, forêts et désertification de l'Afrique tropicale. Société d'Editions Géographiques Maritimes et Coloniales.

Bai, X., Huang, Y., Ren, W., Coyne, M., Jacinthe, P. A., Tao, B., \& Matocha, C. (2019). Responses of soil carbon sequestration to climate-smart agriculture practices: A meta-analysis. Global Change Biology, 25(8), 2591-2606. https://doi.org/10.1111/gcb.14658

Barbosa Neto, M. V., de Araújo, M. D. S. B., de Araújo Filho, J. C., Sampaio, E. V. D. S. B., \& de Almeida, B. G. (2021). Rill and sheet soil erosion estimation in an area undergoing desertification in the Brazilian semi-arid region. Modeling Earth Systems and Environment, 7(2), $1183-1191$.

Barros Corrêa A.C., de Azevêdo Cavalcanti Tavares B., de Lira D. R., da Silva Mutzenberg D., \& de Souza Cavalcanti L.C. (2019). The Semi-arid Domain of the Northeast of Brazil. In: Salgado A., Santos L., Paisani J. (eds) The Physical Geography of Brazil. Geography of the Physical Environment. Springer, Cham.

Barros, K. O., Ribeiro, C. A. A. S., Marcatti, G. E., Lorenzon, A. S., de Castro, N. L. M., Domingues, G. F., \& Dos Santos, A. R. (2018). Markov chains and cellular automata to predict environments subject to desertification. Journal of Environmental Management, 225, $160-167$.

Basso, F., Bove, E., Dumontet, S., Ferrara, A., Pisante, M., Quaranta, G. \& Taberner, M. (2000) Evaluating environmental sensitivity at the basin scale through the use of geographic information systems and remotely sensed data: an example covering the Agri basin - Southern Italy. Catena, 40, 19-35. https://doi.org/10.1016/S0341-8162(99)00062-4

Bezerra, F. G. S., Aguiar, A. P. D., Alvalá, R. C. S., Giarolla, A., Bezerra, K. R. A., Lima, P. V. P. S., \& Arai, E. (2020). Analysis of areas undergoing desertification, using EVI2 multi-temporal data based on MODIS imagery as indicator. Ecological Indicators, 117, 106579.

Biskaborn, B. K., Smith, S. L., Noetzli, J., Matthes, H., Vieira, G., Streletskiy, D. A., \& Lantuit, H. (2019). Permafrost is warming at a global scale. Nature Communications, 10(1), 1-11. https://doi.org/10.1038/s41467-018-08240-4

Briassoulis, H. (2019). Combating land degradation and desertification: The land-use planning quandary. Land, 8, 1-26.

Centro de Gestão e Estudos Estratégicos - CGEE. 2016. Desertificação, degradação da terra e secas no Brasil. Brasília. 252p.

Chen, L., Fortier, D., McKenzie, J. M., \& Sliger, M. (2020). Impact of heat advection on the thermal regime of roads built on permafrost. Hydrological Processes, 34(7), 1647-1664.

Cheng, G., \& Wu, T. (2007). Responses of permafrost to climate change and their environmental significance, Qinghai-Tibet Plateau. Journal of Geophysical Research: Earth Surface, 112(F2). https://doi.org/10.1029/2006JF000631

Conti, J. B. (2008). O conceito de desertificação. In: Climatologia e estudos da paisagem. Rio Claro, 3, p. 39.

Costa, M. S., Oliveira-Júnior, J. F., Santos, P. J., Correia Filho, W. L. F., Gois, G., Blanco, C. J. C., Teodoro, P. E., Silva Junior, C. A., Santiago, B. D., Souza, E. O., Jardim, A. M. R. F. (2021). Rainfall extremes and drought in Northeast Brazil and its relationship with El Niño-Southern Oscillation. International Journal of Climatology, 41, E2111-E2135. https://doi.org/10.1002/joc.6835

Crowther, T. W., Todd-Brown, K. E., Rowe, C. W., Wieder, W. R., Carey, J. C., Machmuller, M. B., \& Bradford, M. A. (2016). Quantifying global soil carbon losses in response to warming. Nature, 540(7631), 104-108. https://doi.org/10.1038/nature20150

Ding, H., \& Xingming, H. (2021). Spatiotemporal change and drivers analysis of desertification in the arid region of northwest China based on geographic detector. Environmental Challenges, 4, 100082.

Fathizad, H., Ardakani, M. A. H., Mehrjardi, R. T., \& Sodaiezadeh, H. (2018). Evaluating desertification using remote sensing technique and object-oriented classification algorithm in the Iranian central desert. Journal of African Earth Sciences, 145, 115-130.

Feng, D., Huang, X., Liu, Y., \& Willison, J. M. (2016). Growth and changes of endogenous hormones of mulberry roots in a simulated rocky desertification area. Environmental Science and Pollution Research, 23(11), 11171-11180. https://doi.org/10.1007/s11356-016-6315-z

Feng, K., Wang, T., Liu, S., Yan, C., Kang, W., Chen, X., \& Guo, Z. (2021). Path analysis model to identify and analyse the causes of aeolian desertification in Mu Us Sandy Land, China. Ecological Indicators, 124, 107386.

Fernandes, J. D., Dantas, A. J. (2021). Desertificação no Nordeste: uma aproximação sobre o fenômeno do Rio Grande Norte. Holos, 3 , 147. 
Ferreira, T. R., Di Pace, T. F., Silva, B. B., \& Delgado, J. R. (2017). Identification of desertification-sensitive areas in the Brazilian northeast through vegetation indices. Engenharia Agricola, 37, 1190-1202.

Gardufio, M. A. (1977). Technology and Desertification, in UNCOD, 319-448.

Gebru, B. M., Lee, W. K., Khamzina, A., Wang, S. W., Cha, S., Song, C., \& Lamchin, M. (2021). Spatiotemporal multi-index analysis of desertification in dry Afromontane forests of northern Ethiopia. Environment, Development and Sustainability, 1-28. https://doi.org/10.1007/s10668-020-00587-3

Glantz, M. H. (1977). Desertification, Environmental Degradation in and around Arid Lands. Westview Press, 346.

Herb, W. R., Janke, B., Mohseni, O., \& Stefan, H. G. (2008). Ground surface temperature simulation for different land covers. Journal of Hydrology, 356(3-4), 327-343. https://doi.org/10.1016/j.jhydrol.2008.04.020

Jardim, A. M. R. F., Silva, J. R. I., Leite, M. L. M. V., Teixeira, V. I., Morato, R. P., Araújo Júnior, G. N., Silva, T. G. F. (2018). Symbiotic interaction in forage crop cultivations: A review. Amazonian Journal of Plant Research, 2, 149-160. https://doi.org/10.26545/ajpr.2018.b00019x

Jardim, A. M. R. F., Silva, M. V., Silva, A. R., Santos, A., Pandorfi, H., Oliveira-Junior, J. F., Lima, J. L. M. P., Souza, L. S. B., Araujo Junior, G. N., Lopes, P. M. O., Moura, G. B. A., \& Silva, T. G. F. (2021a). Spatiotemporal climatic analysis in Pernambuco State, Northeast Brazil. Journal of Atmospheric and Solar-Terrestrial Physics, 223, 105733. https://doi.org/10.1016/j.jastp.2021.105733

Jardim, A. M. R. F., Silva, T. G. F., Souza, L. S. B., Araújo Júnior, G. do N., Alves, H. K. M. N., Souza, M. de S., Araújo, G. G. L., \& Moura, M. S. B. (2021b). Intercropping forage cactus and sorghum in a semi-arid environment improves biological efficiency and competitive ability through interspecific complementarity. Journal of Arid Environments, 188. https://doi.org/10.1016/j.jaridenv.2021.104464

Jardim, A. M. R. F., Souza, L. S. B., Alves, C. P., Araújo, J. F. N., Souza, C. A. A., Pinheiro, A. G., Araújo, G. G. L., Campos, F. S., Tabosa, J. N., \& Silva, T. G. F. (2021c). Intercropping forage cactus with sorghum affects the morphophysiology and phenology of forage cactus. African Journal of Range and Forage Science, 38:1-12. https://doi.org/10.2989/10220119.2021.1949749

Jardim, A. M. R. F., Santos, H. R. B., Alves, H. K. M. N., Ferreira-Silva, S. L., Souza, L. S. B., Araújo Júnior, G. N., Souza, M. S., Araújo, G. G. L., Souza, C. A. A., \& Silva, T. G. F. (2021d). Genotypic differences relative photochemical activity, inorganic and organic solutes and yield performance in clones of the forage cactus under semi-arid environment. Plant Physiology and Biochemistry, 162, 421-430. https://doi.org/10.1016/j.plaphy.2021.03.011

Jardim, A. M. R. F., Araújo Júnior, G. D. N., Silva, M. J., Morais, J. E. F., \& Silva, T. G. F. (2017). Estimativas de perda de solo por erosão hídrica para o município de Serra Talhada, PE. Journal of Environmental Analysis and Progress, 186-193. https://doi.org/10.24221/jeap.2.3.2017.1416.186-193

Joseph, O., Gbenga, A. E., \& Langyit, D. G. (2018). Desertification risk analysis and assessment in Northern Nigeria. Remote Sensing Applications: Society and Environment, 11, 70-82.

Karavitis, C. A., Tsesmelisa, D. E., Oikonomoub, P. D., Kairisa, O., Constantinos Kosmasa, C., Fassoulia, R. C., Hesseld, R., Jettene, V., Moustakasa, N., Todorovica, B., Skondrasa, N. A., Vasilakoua, C. G., Alexandrisa, S., Kolokythaf, E., Stamatakosa, D. V., Stricevicg, R., Chatzigeorgiadish, E., Brandti, J., Nicky Geesoni, N., \& Giovanni Quaranta, G. (2020). A desertification risk assessment decision support tool (DRAST). Catena, 187, 104413.

Karnieli, A., Qin, Z., Wu, B., Panov, N., \& Yan, F. (2014). Spatio-temporal dynamics of land-use and land-cover in the Mu Us sandy land, China, using the change vector analysis technique. Remote Sensing, 6(10), 9316-9339.

Kosmas, C., Ferrara, A., Briasouli, H., \& Imeson, A. (1999). The MEDALUS project: Mediterranean desertification and land use. Manual on key indicators of desertification and mapping environmentally sensitive areas to desertification European commission. Luxembourg: Office for Official Pubolications of the European Communities, 94p.

Kosmas, C., Kairis, Or., Karavitis, C. H., Ritsema, C., Salvati, L., Acikalin,S., Alcala', M., Alfama, P., Atlhopheng, J., Barrera, J., Belgacem, A., Sole’-Benet, A., Brito, J., Chaker, M., Chanda, R., Coelho, C., Darkoh, M., Diamantis, I., Ermolaeva, O., Fassouli, M., Fei, W., Feng, J., Fernandez, F., Ferreira, A., Gokceoglu, C., Gonzalez, D., Gungor, H., Hessel, R., Juying, J., Khatteli, H., Khitrov, N., Kounalaki, A., Laouina, A., Lollino, P., Lopes, M., Magole, L.,Medina, L., Mendoza, M., Morais, P., Mulale, K., Ocakoglu, F., Ouessar, M., Ovalle, C., Perez, C., Perkins, J., Pliakas, F., Polemio, M., Pozo, M., Prat, C., Qinke, Y., Ramos, A., Ramos, J., Riquelme, J., Romanenkov, V., Rui, L., Santaloia, F., Sebego, R., Sghaier, M., Silva, N., Sizemskaya, M., Soares, J., Sonmez, H., Taamallah, M., Tezcan, T., Torri, D., Ungaro, F., Valente, S., Vente, J., Zagal, E., Zeiliguer, A., Zhonging, W., \& Ziogas, A. (2013). Evaluation and Selection of Indicators for Land Degradation and Desertification Monitoring: Methodological Approach. Environmental Management, 54, 951-970.

Lamchin, M., Lee, W. K., Jeon, S. W., Lee, J. Y., Song, C., Piao, D., \& Navaandorj, I. (2017). Correlation between desertification and environmental variables using remote sensing techniques in Hogno Khaan, Mongolia. Sustainability, 9(4), 581. https://doi.org/10.3390/su9040581

Lavee, H., Imeson, A. C., \& Sarah, P. (1998). The impact of climate change on geomorphology and desertification along a Mediterranean-arid transect. Land Degradation \& Development, 9(5), 407-422. https://doi.org/10.1002/(SICI)1099-145X(199809/10)9:5\%3C407::AID-LDR302\%3E3.0.CO;2-6

Le Houerou, H. N. (1977). Man and desertization in the Mediterranean region. Ambio, 6: 363-365.

Lin, H., Chen, Y., Song, Q., Fu, P., Cleverly, J., Magliulo, V., Law, B., Gough, C. M., Hörtnagl, L., Di Gennaro, F., Matteucci, G., Montagnani, L., Duce, P., Shao, C., Kato, T., Bonal, D., Paul-Limoges, E., Beringer, J., Grace, J., \& Fan, Z. (2017). Quantifying deforestation and forest degradation with thermal response. Science of the Total Environment, 607-608, 1286-1292.

Lin, M. L., Chen, C. W., Wang, Q. B., Cao, Y., Shih, J. Y., Lee, Y. T., \& Wang, S. (2009). Fuzzy model-based assessment and monitoring of desertification using MODIS satellite imagery. Engineering Computations, 745-760.

Liu, A. (2005). Monitoring Desertication in Arid and Semi-arid Areas of China with NOAA-AVHRR and MODIS Data. International Geoscience and Remote Sensing Symposium, 4, pp. 2362-2364.

Lopes, L. S. D. O., \& Soares, R. C. (2016). Suscetibilidade à desertificação das terras secas de Gilbués (Estado do Piauí) e Cabrobó (Estado do Pernambuco), nordeste do Brasil. Revista Geográfica de América Central, 1(56), 307-323. http://dx.doi.org/10.15359/rgac.1-56.13 
Mariano, D. A., dos Santos, C. A., Wardlow, B. D., Anderson, M. C., Schiltmeyer, A. V., Tadesse, T., \& Svoboda, M. D. (2018). Use of remote sensing indicators to assess effects of drought and human-induced land degradation on ecosystem health in Northeastern Brazil. Remote Sensing of Environment, 213, $129-143$.

Matallo Junior, H. (2001). Indicadores de Desertificação: histórico e perspectivas. UNESCO, 80p.

Nippert, J. B., Ocheltree, T. W., Orozco, G. L., Ratajczak, Z., Ling, B., \& Skibbe, A. M. (2013). Evidence of physiological decoupling from grassland ecosystem drivers by an encroaching woody shrub. PLoS One, 8(12), e81630. https://doi.org/10.1371/journal.pone.0081630

Niu, F., Gao, Z., Lin, Z., Luo, J., \& Fan, X. (2019). Vegetation influence on the soil hydrological regime in permafrost regions of the Qinghai-Tibet Plateau, China. Geoderma, 354, 113892.

Nolêto, T. M. S. D. J. (2005). Suscetibilidade geoambiental das terras secas da microrregião de Sobral/CE à desertificação. p. 144.

Oikonomou, P. D., Tsesmelis, E. D., Waskom, M. R., Grigg, N. S., Karavítis, C. A. (2019). Enhancing the standardized drought vulnerability index by integrating spatiotemporal information from satellite and in situ data. Journal of Hydrology, 569, 265-277.

Olsson, L., Eklundh, L., \& Ardö, J. (2005). A recent greening of the Sahel-trends, patterns and potential causes. Journal of Arid Environments, 63(3), 556566.

Oliveira-Júnior, J. F., de Gois, G., de Lima Silva, I. J., de Oliveira Souza, E., Jardim, A. M. R. F., Silva, M. V., \& Jamjareegulgarn, P. (2021). Wet and dry periods in the state of Alagoas (Northeast Brazil) via Standardized Precipitation Index. Journal of Atmospheric and Solar-Terrestrial Physics, 224, 105746. https://doi.org/10.1016/j.jastp.2021.105746

Pan, J., \& Li, T. (2013). Extracting desertification from Landsat TM imagery based on spectral mixture analysis and Albedo-Vegetation feature space. Natural Hazards, 68(2), 915-927.

Pereira Neto, M. (2016). Predisposição à desertificação no núcleo Seridó (RN - Brasil): Geoecologiade Paisagens Semiárida, 197p.

Perez-Marin, A. M., Cavalcante, A. M. B., Medeiros, S. S., Tinôco, L. B. M., \& Salcedo, I. H. (2012). Núcleos de desertificação no semiárido brasileiro: ocorrência natural ou antrópica? Parcerias Estratégicas, 17, 87-106.

Pinto, R. M. S., Carvalho, V. C., \& Alvalá, R. C. S. (2009). Mapas de variabilidade temporal do uso e cobertura da terra do núcleo de desertificação de Irauçuba (CE) para utilização em modelos meteorológicos. Anais... XIV Simpósio Brasileiro de Sensoriamento Remoto, Natal. XIV SBSR.

Pinheiro, A. G., Jardim, A. M. R. F., Montenegro, A. A. A., Silva, T. G. F., \& Silva, J. R. (2021). Characterization of alluvial soil hydrodynamics in the upper Ipanema river basin using the Beerkan method. DYNA, 88(218), 178-184. https://doi.org/10.15446/dyna.v88n218.91077

Prăvălie, R., Patriche, C., Săvulescua, I., Sîrodoevc, I., Bandoca, G., \& Sfîcă, L. (2020). Spatial assessment of land sensitivity to degradation across Romania. A quantitative approach based on the modified MEDALUS methodology. Catena, 187, 104407.

Qian, C., Qiang, H., Wang, F., \& Li, M. (2021). Optimization of Rocky Desertification Classification Model Based on Vegetation Type and Seasonal Characteristic. Remote Sensing, 13(15), 2935. https://doi.org/10.3390/rs13152935

Reining, P. (1978). Handbook on Desertification Indicators Based on the Science Associations' Nairobi Seminar on Desertification, American Association for the Advancement of Science, 141.

Retallack, G. J. (1991). Soils of the past: an introduction to paleopedology. Harper Collins Academic.

Reynolds, J. F., Grainger, A., Stafford Smith, D. M., Bastin, G., Garcia-Barrios, L., Fernández, R. J., \& Zdruli, P. (2011). Scientific concepts for an integrated analysis of desertification. Land Degradation \& Development, 22(2), 166-183. https://doi.org/10.1002/ldr.1104

Rodrigues, V. (1992). Avaliação do quadro da Desertificação no Nordeste do Brasil: Diagnósticos e perspectivas. Conferência Internacional sobre Impacto das Variações Climáticas e Desenvolvimento Sustentável em Regiões Semiáridas. Fortaleza.

Rudorff, C. D. M., Rizzi, R., Rudorff, B. F. T., Sugawara, L. M., \& Vieira, C. A O. (2007). Superfícies de resposta espectro-temporal de imagens do sensor MODIS para classificação de área de soja no Estado do Rio Grande do Sul. Ciência Rural, 37, 118-125.

Sá, C. S. B., Shiosaki, R. K., dos Santos, A. M., \& da Silva Campos, M. A. (2021). Salinization causes abrupt reduction in soil nematode abundance in the Caatinga area of the Submedio San Francisco Valley, Brazilian semiarid region. Pedobiologia, 85, 150729.

Sá, I. B., \& Angelotti, F. (2008). A degradação ambiental e a situação atual dos recursos naturais no Semi-Árido. Mudanças Climáticas e Desertificação no Semi-árido Brasileiro, 53-76.

Sá, I. B., \& Angelotti, F. (2009). Degradação ambiental e desertificação no semi-árido brasileiro. In: Angelotti F, Sá IB, Menezes EA, Pellegrino GQ (eds) Mudanças climáticas e desertificação no semi-árido brasileiro. Embrapa Semiárdo, 59-8.

Sales, M. C. L. (2002). Evolução dos estudos de desertificação no nordeste brasileiro. GEOUSP Espaço e Tempo, 6, 115-126. https://doi.org/10.11606/issn.2179-0892.geousp.2002.123650

Sales, M. C. L. (2006). O panorama da desertificação no Brasil. In: Moreira, E. (org.) Agricultura familiar e desertificação. João Pessoa: Editora Universitária da UFPB.

Salvati, L., Bajocco, S., Ceccarelli, T., Zitti, M., \& Perini, L. (2011). Towards a process-based evaluation of land vulnerability to soil degradation in Italy. Ecological Indicators, 11, 1216-1227. 
Santos, A., Lopes, P. M. O., da Silva, M. V., Jardim, A. M. R. F., de Albuquerque Moura, G. B., Fernandes, G. S. T., \& de Oliveira-Júnior, J. F. (2020). Causes and consequences of seasonal changes in the water flow of the São Francisco river in the semiarid of Brazil. Environmental and Sustainability Indicators, 8, 100084. https://doi.org/10.1016/j.indic.2020.100084

Salvati, L., Zitti, M., \& Perini, L. (2016). Fifty Years on: Long-term Patterns of Land Sensitivity to Desertification in Italy. Land Degradation and Development, 27, 97-107.

Sepehri, A., Sarrafzadeh, M. H., \& Avateffazeli, M. (2020). Interaction between Chlorella vulgaris and nitrifying-enriched activated sludge in the treatment of wastewater with low C/N ratio. Journal of Cleaner Production, 247, 119164. https://doi.org/10.1016/j.jclepro.2019.119164

Shaoling, W., Huijun, J., Shuxun, L., \& Lin, Z. (2000). Permafrost degradation on the Qinghai-Tibet Plateau and its environmental impacts. Permafrost and Periglacial Processes, 11(1), 43-53.

Silva, A. K. O., \& Silva, H. P. B. (2015). O Processo De Desertificação E Seus Impactos Sobre Os Recursos Naturais E Sociais No Município De Cabrobó Pernambuco - Brasil. Revista Eletrônica de Humanidades do Curso de Ciências Sociais da UNIFAP, 8, $203-215$.

Silva, M. V., Pandorfi, H., Lopes, P. M. O., da Silva, J. L. B., de Almeida, G. L. P., de Oliveira Silva, D. A., \& Jardim, A. M. R. F. (2020a). Pilot monitoring of caatinga spatial-temporal dynamics through the action of agriculture and livestock in the Brazilian semiarid. Remote Sensing Applications: Society and Environment, 19, 100353. https://doi.org/10.1016/j.rsase.2020.100353

Silva, G. I. N., Jardim, A. M. D. R. F., Alves, H. K. M. N., de Sá Souza, M., de Souza, C. A. A., Júnior, G. D. N. A., \& da Silva, T. G. F. (2020b). Cultivo de plantas forrageiras de apelo regional para o Semiárido brasileiro sob a perspectiva de técnicas agrometeorológicas para melhoria da resiliência: uma revisão. Research, Society and Development, 9(10), e1449108291. https://doi.org/10.33448/rsd-v9i10.8291

Silva, H. P., Ribeiro, M. R., Melo, I. C. L. G. H., \& Bezerra S. A. L. (2011). Utilização de técnicas de sensoriamento remoto na identificação de áreas sob risco de desertificação no estado de Pernambuco. XV Simpósio Brasileiro de Sensoriamento Remoto - SBSR, (1), 9144-9151.

Silva, M. V., Pandorfi, H., Jardim, A. M. R. F., Oliveira-Júnior, J. F., Divincula, J. S., Giongo, P. R., \& Lopes, P. M. O. (2021a). Spatial modeling of rainfall patterns and groundwater on the coast of northeastern Brazil. Urban Climate, 38, 100911. https://doi.org/10.1016/j.uclim.2021.100911

Silva, M. V., Pandorfi, H., de Almeida, G. L. P., de Lima, R. P., dos Santos, A., Jardim, A. M. R. F., \& da Silva, D. C. (2021b). Spatio-temporal monitoring of soil and plant indicators under forage cactus cultivation by geoprocessing in Brazilian semi-arid region. Journal of South American Earth Sciences, 107, 103155. https://doi.org/10.1016/j.jsames.2021.103155

Sommer, S., Zucca, C., Grainger, A., Cherlet, M. Zougmore, R., Sokona, Y., Hill, J., Della, R., Roehrig, J., \& Wang, G. (2011). Application of indicator systems for monitoring and assessment of desertification from national to global scales. Land Degradation and Development, 22, $184-197$.

Sousa, R. F., Barbosa, M. P., Morais Neto, J. M., \& Fernandes, M. F. (2007). Estudo do processo de desertificação e das vulnerabilidades do município de Cabaceiras - Paraíba. Engenharia Ambiental, 4, 89-102.

Spinoni, J., Vogt, J., Naumann, G., Carrao, H., \& Barbosa, P. (2015). Towards identifying areas at climatological risk of desertification using the KöppenGeiger classification and FAO aridity index. International Journal of Climatology, 35(9), 2210-2222.

Stebbing, E. P. (1935). The Encroaching Sahara. Geographical Journal, 86, 509-510.

Teufel, B., \& Sushama, L. (2019). Abrupt changes across the Arctic permafrost region endanger northern development. Nature Climate Change, 9(11), 858862.

Tomasella, J., Vieira, R. M. S. P., Barbosa, A. A., Rodriguez, D. A., de Oliveira Santana, M., \& Sestini, M. F. (2018). Desertification trends in the Northeast of Brazil over the period 2000-2016. International Journal of Applied Earth Observation and Geoinformation, 73, 197-206. https://doi.org/10.1016/j.jag.2018.06.012

Tomasella, J., Vieira, R. M. S. P., Barbosa, A. A., Rodriguez, D. A., de Oliveira Santana, M., \& Sestini, M. F. (2018). Desertification trends in the Northeast of Brazil over the period 2000-2016. International Journal of Applied Earth Observation and Geoinformation, 73, $197-206$.

UNCCD, Desertification Secretariat. 1995. Down to Earth. A simplified guide to the Convention to Combat Desertification, why it is necessary and what is important and different about it. Bonn: UNCCD.

United Nations Environment Programme - UNEP. (1991). Status of desertification and implementation of the United Nations Plan of Action to Combat Desertification. Nairóbi: UNEP.

Vasconcelos Sobrinho, J. (1982). Processos de desertificação no Nordeste do Brasil: sua gênese e sua contenção. Recife: Sudene.

Vasconcelos, C. H., \& Novo, E. M. L. M. (2004). Mapeamento do uso e cobertura da terra a partir da segmentação e classificação de imagens-fração solo, sombra e vegetação derivadas do modelo linear de mistura aplicado a dados do sensor TM/Landsat5, na região do reservatório de Tucuruí - PA. Acta Amazonica, 34, 487-493.

Verón, S. R., Blanco, L. J., Texeira, M. A., Irissari, G. N., \& Paruelo, J. M. (2018). Desertification and ecosystem services supply: The case of the Arid Chaco of South America. Journal of Arid Environments, 159, 66-74.

Verstraete, M. M. (1986). Defining desertification: A review. Climatic Change, 9, 5-18.

Vieira, N. M. (1978). Estudo geomorfológico das voçorocas de Franca - SP. Tese (Doutorado em História) - Instituto de História e Serviço Social, Universidade Estadual Paulista, Franca, 225. 
Research, Society and Development, v. 10, n. 11, e585101119950, 2021

(CC BY 4.0) | ISSN 2525-3409 | DOI: http://dx.doi.org/10.33448/rsd-v10i11.19950

Vieira, R. M. D. S. P., Sestini, M. F., Tomasella, J., Marchezini, V., Pereira, G. R., Barbosa, A. A., \& Ometto, J. P. H. B. (2020). Characterizing spatiotemporal patterns of social vulnerability to droughts, degradation and desertification in the Brazilian northeast. Environmental and Sustainability Indicators, 5, 100016.

Vieira, R. M. S. P., Sestini, M. F., Tomasella, J., Rodriguez, D. A., Barbosa, A. A., Campello, F. C. B., Santana, M. O., Ometto, J. P. H. B., \& Valles, G. F. (2016). Avaliação do impacto da desertificação no ambiente e na população do Semiárido brasileiro por meio de uma base de dados geográficos. Sustentabilidade em Debate, 7, 52-68.

Vieira, R. M. S. P., Tomasella, J., Alvalá, R. C. S., Sestini, M. F., Affonson, A. G., Rodriguez, D. A., Barbosa, A. A., Cunha, A. P. M. A., Valles, G. F., Crepani, E., Oliveira, S. B. P., Souza, M. S. B., Campello, F. C. B., \& Santana, M. O. (2015). Identifying areas susceptible to desertification in the Brazilian northeast. Solid Earth, 6, 347-360.

Wei, H., Wang, J., Cheng, K., Li, G., Ochir, A., Davaasuren, D., \& Chonokhuu, S. (2018). Desertification information extraction based on feature space combinations on the Mongolian plateau. Remote Sensing, 10(10), 1614. https://doi.org/10.3390/rs10101614

Wu, P., Liang, S., Wang, X. S., Feng, Y., \& McKenzie, J. M. (2018). A new assessment of hydrological change in the source region of the Yellow River. Water, 10(7), 877. https://doi.org/10.3390/w10070877

Xavier Junior, O. S., Jardim, A. M. R. F., de Souza, L. S. B., de Souza, C. A. A., da Cruz Neto, J. F., Júnior, G. D. N. A., \& da Silva, T. G. F. (2021). Utilização de biofertilizantes alternativos no cultivo de palma forrageira: Uma revisão. Research, Society and Development, 10(6), e46110616024e46110616024.

Xiao, J., Tateishi, R., \& Bayaer, W. (2006). Development of topsoil grain size index for monitoring desertification in arid land using remote sensing. International Journal of Remote Sensing, 27, 2411-2422.

Xie, S., Qu, J., Lai, Y., Xu, X., \& Pang, Y. (2015). Key evidence of the role of desertification in protecting the underlying permafrost in the Qinghai-Tibet Plateau. Scientific Reports, 5(1), 1-8.

Xue, J., Gui, D., Lei, J., Sum, H., Zeng, F., Mao, D., Jim, Q., \& Liu, Y. (2019). Oasification: An unable evasive process in fighting against desertification for the sustainable development of arid and semiarid regions of China. Catena, 179, 197-209.

Yang, M., Nelson, F. E., Shiklomanov, N. I., Guo, D., \& Wan, G. (2010). Permafrost degradation and its environmental effects on the Tibetan Plateau: A review of recent research. Earth-Science Reviews, 103(1-2), 31-44. https://doi.org/10.1016/j.earscirev.2010.07.002 